\title{
Acoustic Time Measurement Algorithms of Ultrasound Based on Integer-Decimal Detection
}

\author{
Jiaming Zeng ${ }^{1}$, Qiang Xiang ${ }^{*}$, Lie $\mathrm{Guo}^{2}$, Youan $\mathrm{Shi}^{3}$ and Wei Chang ${ }^{3}$ \\ ${ }^{1}$ College of Electrical Information Engineering, Southwest Minzu University, Chengdu, Sichuan, China \\ ${ }^{2}$ College of Computer Technology and Engineering, University of Electronic Science and Technology of China, \\ Chengdu, Sichuan, China \\ ${ }^{3}$ China Aerodynamic Research and Development Center, Mianyang, Sichuan, China \\ Email: xqiang_0426@163.com
}

\begin{abstract}
Ultrasound measurement technology can be used in nondestructive testing, temperature measurement, ranging and other industrial fields. These technologies all depend on the measurement of ultrasonic wave speed or sound time. At present, the commonly used ultrasonic time measurement methods are direct time difference method and phase difference method, but both of them have certain limitations. In this paper, an algorithm for high precision ultrasonic time measurement is presented. In this algorithm, zynq-7000 FPGA is used as the control core of the system hardware circuit, and the signal generator module is used to generate the ultrasonic signal. The time difference and phase difference between the received and received signals are measured to determine the ultrasonic transit time. The experimental results show that the system can accurately measure the time, the phase resolution is 0.007 degrees, and the time accuracy is better than 1.0ns (nanoseconds).
\end{abstract}

Keywords: Ultrasound time, direct time difference, phase difference, integer-decimal detection, FPGA

\section{Introduction}

Time interval is an important index of measurement technology, which is of great significance in industrial production and national defense construction. Especially high-precision time interval measurement technology, is not only widely used in positioning, communications, medical and other fields, but also plays a decisive role in the military.

The measurement of sound speed actually includes the measurement of sound time $t$ and sound path $L$. Usually the sound path is a known quantity, so that the measurement of sound speed is converted to the measurement of sound time. The sound speed is as follows: $c=L / t$.

The commonly used time measurement methods include direct time difference method, phase difference method, analog interpolation method and time-amplitude conversion method. The principle and operation of direct time difference method are simple, the circuit structure is simple and the measurement is convenient. However, its accuracy is greatly affected by the clock frequency of the counter. For example, the time accuracy of $100 \mathrm{MHz}$ clock frequency measurement commonly used in the market can only reach about $0.01 \mu \mathrm{s}(1 / 100 \mathrm{MHz})$, which is very difficult for many tests that need to reach ns level or even higher accuracy requirements.

The phase difference method calculates the phase difference of the receiving and receiving signals, and uses the relationship between the phase difference and the period to calculate the time difference. Suppose that the frequency of sound wave is $2 \mathrm{MHz}$ and 12-bit A/D converter is used, so that the theoretical and practical detection accuracy can reach $0.122 \mathrm{~ns}$, and the detection accuracy is relatively high. However, the disadvantage is that it is impossible to judge whether the phase difference exceeds 360 degrees, which results in a rather narrow measurement range.

The analog interpolation method has obvious nonlinearity, large error, long conversion time and extremely complex circuit design. The time-amplitude conversion method converts time measurement into voltage measurement, which has the advantages of easy debugging, large non-linear error and poor stability. Moreover, the time-amplitude converter is analog circuit, and its fabrication process and debugging are complex. 
Combined with the above analysis, the proposed Integer-Decimal Detection algorithm combines the advantages of direct time difference method and phase difference method, avoiding the shortcomings of the two methods. The direct time difference method is used to measure the whole time, and the phase difference method is used to measure the decimal. The sum of the whole decimal is the high-precision sound time.

In the field of temperature measurement, the measurement of temperature field inside structure is of great significance to quality assurance, process control, safety assessment, etc. It has a wide application background and strong demand in military, energy, machinery, metallurgy and medical fields. At present, the temperature field inside the structure is mainly measured by contact type, that is, by sticking or welding on the surface of the measured object, or by installing sensors in the internal perforation. However, this method has some limitations, such as local temperature change or stress concentration caused by the failure of the perforated structure. Although infrared technology and phosphorescence thermography technology can achieve non-destructive temperature measurement, they are limited to the structural surface.

Ultrasound temperature measurement technology can realize non-destructive and non-contact detection of the temperature field inside the structure. By calibrating the functional relationship between the ultrasonic signal (mostly wave velocity) and the temperature of the medium, the temperature range from 2-20K to $17000 \mathrm{~K}$ can be measured. In addition, the ultrasonic temperature measurement method has the advantages of faster, more accurate and wider application range than the traditional temperature measurement method, so it has a broad application prospect. In some special occasions and extreme conditions where conventional temperature measurement methods cannot be applied, such as adverse thermal protection structures, nuclear reactor under high temperature and strong radiation conditions, rocket launching, plasma chamber, inert gas and other high temperature measurements, the ultrasonic method is also considered as one of the most potential temperature measurement technologies.

Because of the fast propagation speed and short transit time of ultrasound in the medium, it is necessary to measure the propagation of ultrasound in the medium at a very high acquisition frequency in order to identify the temperature field inside the medium. It is pointed out that the acquisition frequency cannot meet the current requirements, so it is necessary to apply the integer-decimal detection technology in this area.

\section{Principle of Integer-Decimal Detection}

As shown in Fig. 1, the propagation time of sound wave is equal to the sum of the integral part int of the propagation time (the integral multiple of the period) and the decimal part odd (the decimal multiple of the period).

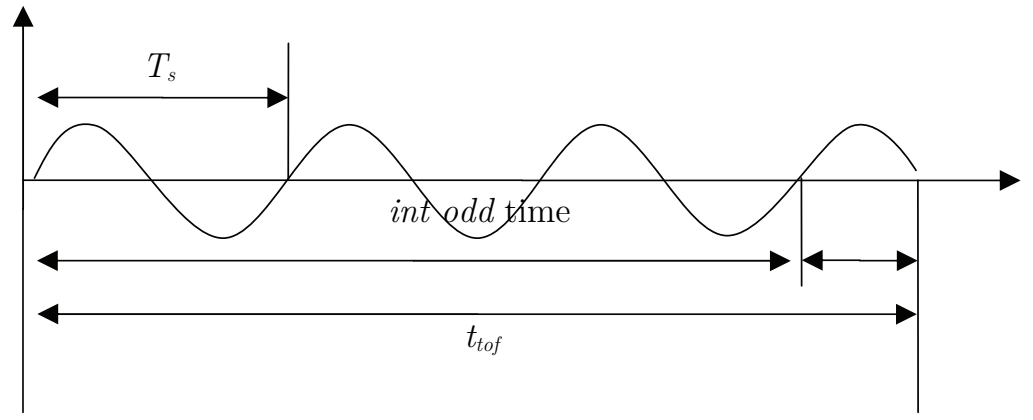

Figure 1. Principle of integer-decimal detection

It is assumed that the excitation waveform satisfies:

$$
u_{s}(t)=U_{s} \cos \left(2 \pi f t+\theta_{0}\right)
$$

In the formula, $U_{s}$ is the amplitude of the waveform, $f$ is the frequency of ultrasound, $\theta_{0}$ is the initial phase. 
The received echo signal satisfies:

$$
u_{r}(t)=U_{r} \cos \left(2 \pi f t+\theta_{r}\right)
$$

In the formula, $U_{r}$ is the receiving waveform amplitude, $\theta_{r}$ is the phase of received wave.

$$
\Delta \theta=\theta_{r}-\theta_{0}=\theta_{N}+\theta_{c}, \quad \theta_{N}=2 \pi f N T_{s}
$$

In the formula, $\theta_{N}$ is the Integer-fold phase difference between received and exicited waves, $\theta_{c}$ is the Non-integer phase difference between received and excited waves, $T_{s}$ is the period of ultrasound, $N$ is the integral period multiple between the excitation wave and the received wave.

Integer multiple period $N$ can be obtained by direct counting method.

$$
N=\operatorname{int}\left(\frac{t_{t o f, 0}}{T_{s}}\right)
$$

The main frequency of ultrasonic wave will not change in the course of propagation. Therefore, the adjacent echo signals are transformed by Fourier transform. By comparing the phase at the main frequency (see formula (5), the non-integer phase difference is obtained.

$$
\theta=\arctan \left(\frac{I_{m}}{R_{m}}\right)
$$

In the formula, $I_{m}$ is the imaginary part corresponding to the main frequency line after Fourier transform, $R_{m}$ is the corresponding real part.

In principle, the period of ultrasonic wave is generally $100 \mathrm{~ns}$, and the measurement of integer multiple period $N$ by direct counting method has very high accuracy. The measurement of non-integer periodic time $\Delta t_{c}$, because of the amplification factor $2 \pi f$, will greatly improve the accuracy, as shown in formula (6).

$$
\theta_{c}=2 \pi f \Delta t_{c}
$$

Formula (4) and Formula (6) are used to obtain the transmission time of Integer-Decimal detection is:

$$
t_{t o f}=N T_{s}+\frac{\theta_{c}}{2 \pi f}
$$

\section{Algorithm Implementation}

According to the above ideas, the hardware schematic diagram of the ultrasomic propagation time measurement system is designed as shown in Fig. 2.

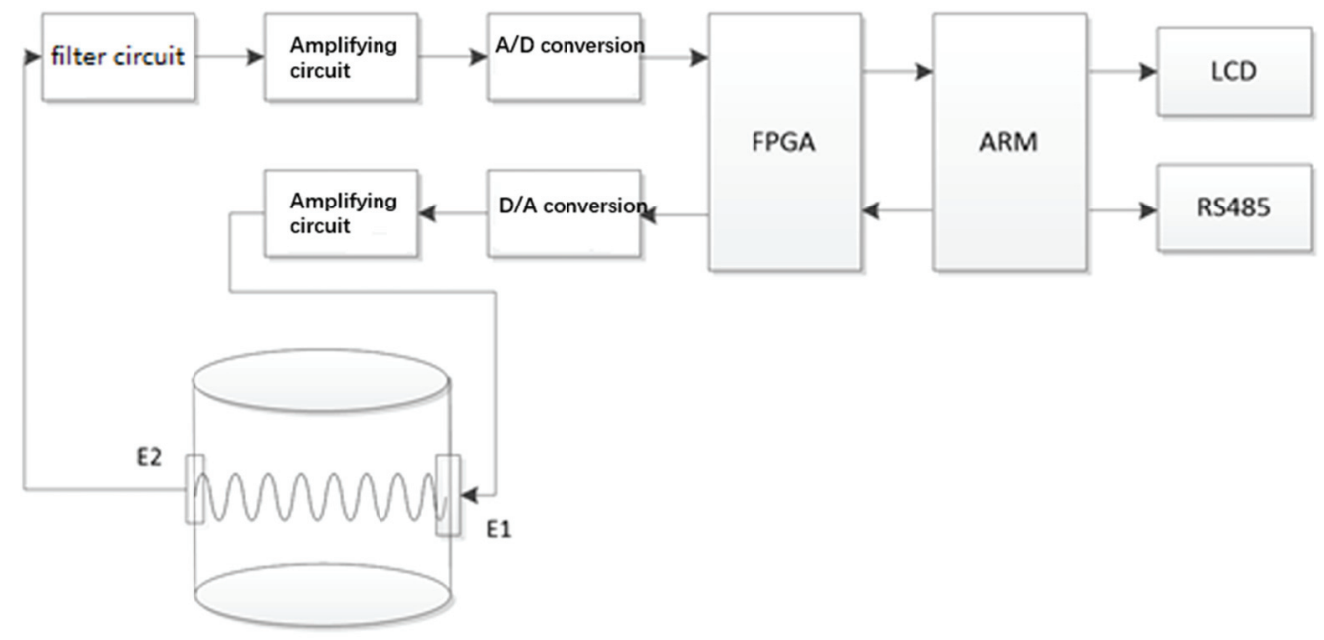

Figure 2. Principle block diagram of ultrasonic temperature measurement 
The work flow of ultrasonic temperature measurement is: ARM controls the FPGA to produce driving signal, and the analog 10 counter of the FPGA starts counting. The driving signal is driven to transmit the transducer E1 by D/A conversion circuit and power amplifier circuit in turn, which makes the driving signal with certain energy transmit the transducer E1 to transmit the ultrasonic wave. The receiving transducer E2 receives the ultrasonic signal emitted from the transmitting transducer E1 and converts it into the ultrasonic echo signal. After being processed by filtering circuit and amplifying circuit, the A/D conversion circuit collects the data and stores the collected data in the memory area of the FPGA. When the data acquisition is completed, the phase difference operation is carried out in the FPGA, and the counter counting is stopped. In the process of ultrasonic wave propagation from transmission to reception, the ultrasonic signal manager has $N$ positive cycles, and there is a zero cycle, which is formally reflected by the phase difference between the received signal and the transmitted signal, that is, the A/D result. The results of counter and phase difference are sent to ARM by AXI GPIO. In ARM, the results of counter are calculated as whole time, and the phase difference is calculated as decimal time. Finally, the combination of the two is the transit time of ultrasound. After the ARM processing is completed, the calculated time is displayed on the LCD or connected to other terminals through RS485 serial port.

In this paper, the main functions of the FPGA are as follows: firstly, the DDS module generates the ultrasonic driving signal; secondly, the received ultrasonic echo signal is sampled at high speed, and the sampled data is stored in the RAM storage area built with the FPGA; thirdly, the sampled data in the RAM storage area is processed to calculate the ultrasonic propagation. Fourthly, the decimal part of the ultrasonic propagation is obtained indirectly by calculating the phase difference between the transmitted and echo signals.

Fig. 3 shows the structure of the FPGA in this study. The internal controller of the FPGA sends the acquisition command, and the signal generator generates the digital sine wave signal under the control of the synchronous circuit. At the same time, the data acquisition circuit starts high-resolution data acquisition, and stores the data of the collected echo signal in the RAM data buffer built in the internal of the FPGA. When the sampling is completed, the controller receives the status information of the sampling, and sends out the instruction to read the data in the RAM data buffer for data reading.

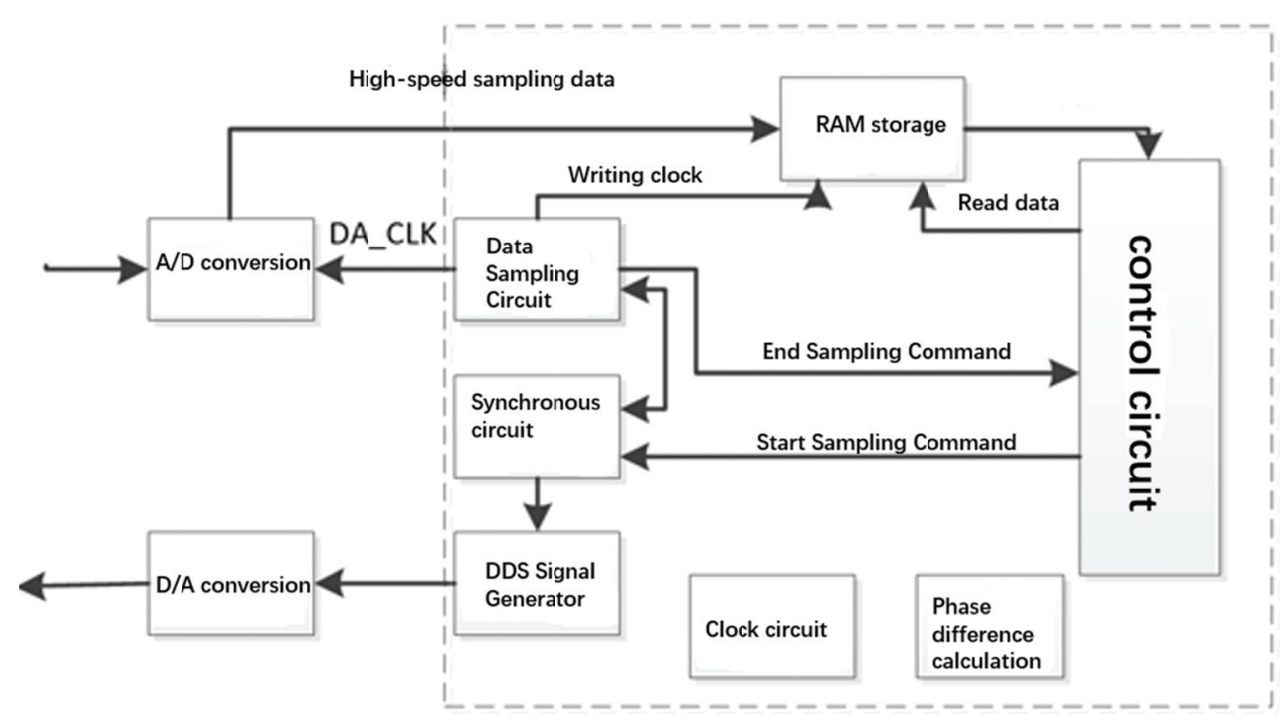

Figure 3. The block diagram of the structure of the FPGA

The integer part time is measured by a modulus 10 counter with a clock cycle of $1 \mathrm{~ns}$. The transit time of receiving and receiving ultrasound is 1ns. Further processing is needed to obtain higher accuracy, that is, the decimal part time of the measurement in this paper. In this paper, the method based on phase difference is used to measure the decimal time. The specific method is shown in Fig. 4. 


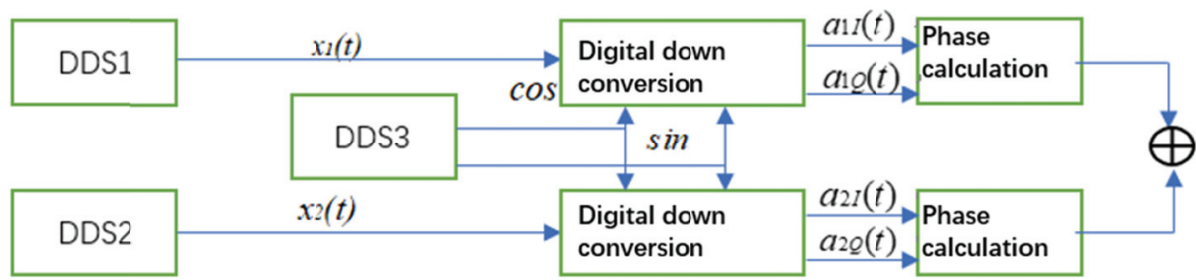

Figure 4. Phase difference implementation block diagram

The DDS Compile IP core is used to generate the sum of two sinusoidal signals $x_{1}(t)$ and $x_{2}(t)$. The frequency is set to $20 \mathrm{MHz}$. The phase of DDS1 is fixed to 0 degrees, and the phasie of DDS2 can be set to 0 to 360 degrees in software. DDS3 generates $x_{3}(t)$ which frequency is 20MHz. $x_{11}(t)$ and $x_{2}(t)$ Separate operate with $x_{3}(t)$ will get the real part $a_{1 I}(t)$ of $x_{1}(t)$, the imaginary part $a_{1 Q}(t)$ of $x_{1}(t)$ and the real part $a_{2 I}(t)$ of $x_{2}(t)$, the imaginary part $a_{2 Q}(t)$ of $x_{2}(t)$. By formula (5) will get the phases of $x_{1}(t)$ and $x_{2}(t)$. The phase difference $\theta_{c}$ is obtained by subtracting the real part and the imaginary part. Then the decimal part of sound time $\Delta t_{c}$ is obtained by formula (6).

\section{Analysis and Discussion}

The results of simulation in vivado by this method are shown in Fig. 5.
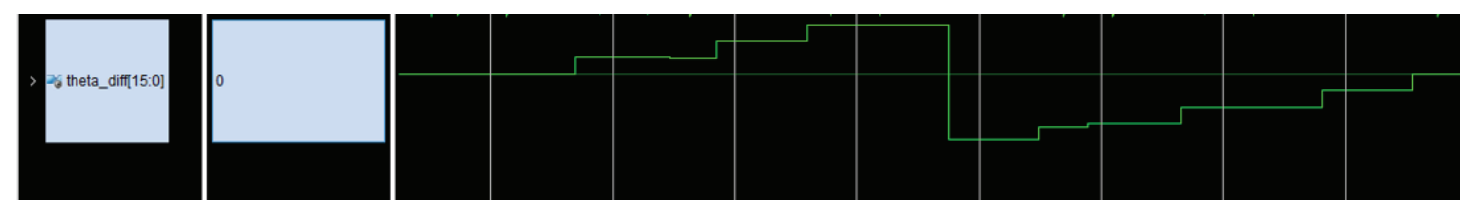

Figure 5. Simulation of phase difference method in vivado

In the aspect of integer measurement, this paper uses the modulus 10 counter, the clock cycle is $1 \mathrm{~ns}$, so that the integer part of the measured results can be accurate to 1ns, and the accuracy of the clock cycle can be adjusted according to the actual situation. In the measurement of decimal part, a variable theta_diff [15:0] is set as the measured phase difference. In this paper, theta_diff [15:0] is a 16-bit radian rad, of which 3-bit integer and 13-bit decimal. Therefore, the radian is worth calculating as follows:

$$
\text { theta_diff }=\text { theta }[15: 0] / 22^{\wedge} 13
$$

For example, theta_diff $[15: 0]$ is $16^{\prime} \mathrm{d} 6434=\left\{3^{\prime} \mathrm{b} 000,13^{\prime} \mathrm{b} 1 \_1001 \_0010\right\}$, then the radian value is: $6434 /(2 \prec 13)=0.7854=45$ degree. Resolving power is $1 \mathrm{rad} / 2^{-13}=57.3$ degree $/ 2 \prec 13=0.007$ degree. Set the frequency of ultrasonic wave to $20 \mathrm{MHz}$, from formula (6) will get $\Delta t_{c}$ 's accuracy is $5.57 \mathrm{e}-10 \mathrm{~s}$.

The accuracy of integer part and decimal part can reach $10 \mathrm{e}-10 \mathrm{~s}$, which can be applied in many highprecision time measurement occasions.

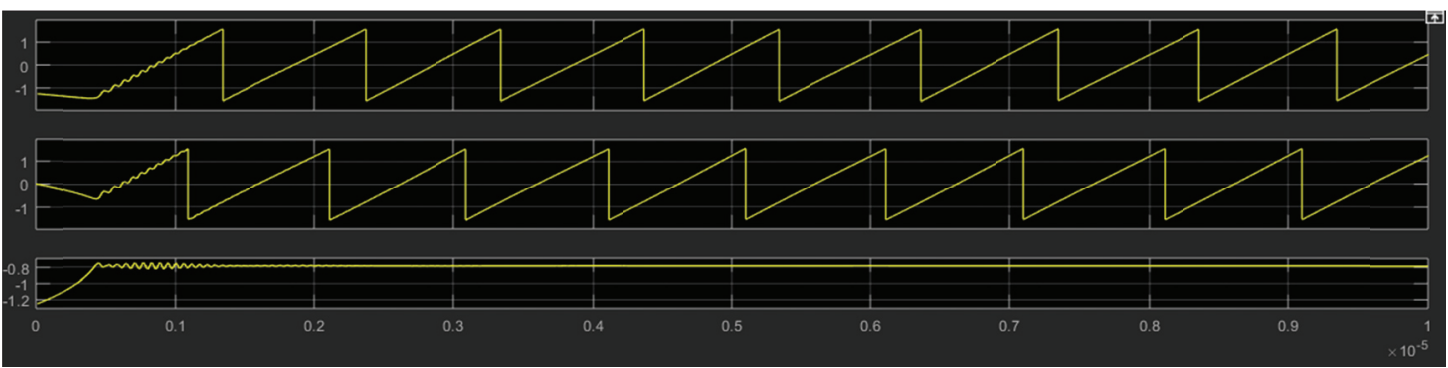

Figure 6. Simulation of phase difference method in MATLAB 
In addition, the phase difference method can also be simulated in MATLAB. The simulation results are shown in Fig. 6.

From the previous discussion, it can be seen that although the direct time difference method has the advantage of wide detection range, it can be used to detect the propagation time of sound. But it has the disadvantage of low detection accuracy. Although the phase difference method has the advantage of high accuracy in detecting sound propagation time, it has the disadvantage of narrow detection range. The integer-decimal detection algorithm based on FPGA can detect propagation time and overcome the shortcomings of both, retaining the advantages of both. The phase resolution is 0.007 degrees, and the accuracy of acoustic time measurement is better than $1 \mathrm{~ns}$. It is easy to integrate, low cost and easy to implement. It is a good detection technology.

Acknowledgments. The work of this paper is supported by the Southwest Minzu University Graduate Innovative Research Project (Master Program CX2019SZ15). A special acknowledgement should give to Southwest Minzu University for its experimental conditions and technical support.

\section{References}

1. Gang Xiong, "Accurate detection of sound propagation time by means of integer-decimal detection technology", Computer measurement and control, 2006,14 (3): 305-306.

2. Xinghong Zhang, "Design of reflective ultrasonic thermometer, Instrument technology and sensors", 2014, 9:1618.

3. Youan Shi, "Research on the ultrasonic detection method of temperature field inside structure", Mianyang: China Aerodynamic Research and Development Center, 2016.

4. Lei Qiu, "Experimental study of ultrasonic thermometer", Chongqing: Chongqing University of Technology, 2015.

5. Ying Lu, "Design of High Precision Time Measurement Platform Based on FPGA", Harbin: Harbin Engineering University, 2013.

6. Shixiong Su, "Research and Implementation of Key Technologies of Ultrasound Guided Wave Thermometry", Taiyuan: Zhongbei University, 2018.

7. Xinghong Zhang, "Design of Precision High Temperature and High Pressure Ultrasound Thermometer", Instrument Technology and Sensors, 2014,11:24-26.

8. Jie Lang, "Implementation of high-precision phase difference measurement algorithm based on FPGA", Modern electronic technology, 2011,34 (21): 28-30.

9. Shengfa Xu, "High precision time interval measurement method based on TDC_GPX", Research and development, 2012, 31 (12): 40-41.

10. A. Tellez, I. Bazan, L. Leijia, A. Vera, "Noise Immunity Analysis of Time Delay Estimation Techniques Used on Ultrasonic Simulated Echoes", 2008 5th International Conference on Electrical Engineering, Computing Science and Automatic Control(CCE 2008):196-200.

11. Shaofeng Dong, Wei Zhou, Baoqiang Du, Changzhe Jiao, "Ultra-high Resolution Phase Difference Measurement Method", IEEE International Frequency Control Symposium, 2012:1-4.

12. Brian J, Tucker, Aaron A, Diaz, Brian A.Eckenrode, "Advanced ultrasonic measurement methodology for noninvasive interrogation and identification of fluids in sealed containers", SPIE Proc,2006,61780K1-61780K12. 